\title{
Selecting Voting Locations for Fun and Profit
}

\author{
Zack Fitzsimmons ${ }^{1}$ and Omer Lev $^{2}$ \\ ${ }^{1}$ College of the Holy Cross, Worcester, MA USA \\ ${ }^{2}$ Ben-Gurion University, Beersheba, Israel \\ zfitzsim@holycross.edu, omerlev@bgu.ac.il
}

\begin{abstract}
While manipulative attacks on elections have been well-studied, only recently has attention turned to attacks that account for geographic information, which are extremely common in the real world. The most well known in the media is gerrymandering, in which district border-lines are changed to increase a party's chance to win, but a different geographical manipulation involves influencing the election by selecting the location of polling places, as many people are not willing to go to any distance to vote. In this paper we initiate the study of this manipulation. We find that while it is easy to manipulate the selection of polling places on the line, it becomes difficult already on the plane or in the case of more than two candidates. Moreover, we show that for more than two candidates the problem is inapproximable. However, we find a few restricted cases on the plane where some algorithms perform well. Finally, we discuss how existing results for standard control actions hold in the geographic setting, consider additional control actions in the geographic setting, and suggest directions for future study.
\end{abstract}

\section{Introduction}

When faced with a set of different options, the use of voting as a method to aggregate multiple agents' preferences on those options is thousands of years old; possibly as old as human society. As human societies grew, people quickly encountered a problem: How to deal with such a large set of voters? One common solution, existing since Athenian times, is the division of political units by location. This is generally done in two ways:

1. Each subdivision makes its choice, and sends representatives to an aggregated assembly. This is the way elections are held in the US and in many Westminster type systems, in which subdivisions (e.g., UK constituencies, Canadian ridings, US districts) select a single representative, and send it to the national parliament.

2. Subdivisions are used for organizational purposes only_-voting (or polling) places divide people according to where they live, but their vote is aggregated with other polling places in the same voting unit. In some countries (e.g., Israel), polling places are the only geographic division, while in others (e.g., US), polling places are the smallest geographic division, but others exist composed of units of polling places (counties, districts, states).

The first case has been explored in the past few years in a series of papers (e.g., [Bachrach et al., 2016; Borodin et al., 2018]), mainly discussing the potential for manipulation by drawing the subdivisions' borders (gerrymandering). The second case of the subdivisions - used for organizational purposes only - has not, to the best of our knowledge, been significantly explored computationally.

The geographic problem in this case is not created by the voting rule, but by the location of the voters and the limited amount of effort each voter is willing or able to expend to cast their vote. Hence, the location of a polling place may have an effect on who votes there. Putting voting locations in convenient places encourages people to vote in the election, while locating them far away discourages participation. This form of control manipulation is already being implemented in various locations [Nichols, 2018], and was recently a flashpoint in Georgia [Reid, 2018].

At its core, the problem is fairly straightforward: Voters are distributed in a geographic space, each having a maximal distance they are willing to travel in order to vote. Assuming $k$ polling places are to be located in a particular area, can putting them in particular places ensure a specified candidate's victory? While we shall use the geographic interpretation throughout this paper, we note that geographic distance is used as a proxy for difficulty of accessing a polling place, and other interpretations are possible. For example, in order to encourage students to submit faculty evaluations, the "distance" becomes how much effort each student needs to put in depending on which platforms are used (e.g., paper forms, web, social networks, etc.).

Our results establish two parameters as key to the computational complexity of this problem. The first is the dimension of the space, with the single dimensional case easier than the plane and beyond. Note that solving the single dimensional problem is not as useless as might be thought at first glancenot only can conceptual, nongeographic, settings be described by it, but even in political settings, voting locations might be located near a central highway or transportation route. 
The second parameter is the number of parties/candidates, with the complexity changing once we leave the two-party system. Naturally, the two-party system is very common in many democracies, but even in settings where there are multiple parties, in many subunits (electoral districts, ridings, or constituencies) there are only two major competitive candidates, making the battle for electoral control mainly one in which two sides participate.

In this paper we introduce a new model considering voter and polling place location, and formalize the associated control problem. We show the complexity difference in the two-party case between the line and higher dimension, and between the two-party and multi-party case. Moreover, we show the multi-party problem is inapproximable. Finally, we discuss how existing results translate to this model and novel results on new control problems which are couched in real-world techniques.

\section{Related Work}

In this paper-as in geographic problems in general-we are particularly interested in control problems. The computational study of electoral control problems was introduced by Bartholdi III et al. [1992]. See Faliszewski and Rothe [2016] for a recent survey of results on electoral control.

We use a distance-bound to model each voter's ability to vote at a polling place. This is similar to the use of prices as used in priced electoral control [Miasko and Faliszewski, 2016], and in the related problem of bribery [Faliszewski et al., 2009], where an agent sets the votes of a subcollection of the voters to ensure a preferred outcome after meeting the price to change their vote.

Selecting a polling place in our model can be seen as adding the group of currently nonparticipating voters within their distance-bound to that polling place to the election. This idea of control by adding groups of voters was previously explored in different ways by Erdélyi et al. [2015]; Bulteau et al. [2015]; Faliszewski et al. [2015]. However, in none of these models can the handling of overlap between groups be adapted to the geography-based groups we consider, and some of the normative properties investigated have no relevance in our setting.

There is also some relation to facility location problems, where the facility locations (which are the candidates) are from a fixed set (rather than everywhere), as explored in Feldman et al. [2016]. Note that many settings explored in this research direction tend to be on the one-dimensional line.

More closely related to our line of work are geographic manipulations of voting districts, commonly referred to as gerrymandering. The theoretical bounds on the influence of gerrymandering on the outcome of an election were established in Bachrach et al. [2016], and complexity results were shown in Lewenberg et al. [2017]; Cohen-Zemach et al. [2018], focusing on graphs. We note that while Lewenberg et al. [2017] did not formally prove a greedy algorithm is an approximation algorithm for the gerrymandering problem, they did use it, de facto, as an algorithm to solve practical cases of gerrymandering (Pegden et al. [2017] suggested a different way to tackle gerrymandering, based on cake-cutting ideas). Borodin et al. [2018] investigated how geographic spread affects gerrymandering ability, and there is a line of research on allowing agents to move between districts [Bervoets and Merlin, 2012; van Bevern et al., 2015; Lev and Lewenberg, 2019].

\section{Preliminaries}

An election consists of a set of candidates $C$ and a collection of voters $V$ where each voter has a corresponding vote or preference order that strictly ranks the candidates in $C$ from most to least preferred. An election system $\mathcal{E}$ is a mapping from an election to a set of winning candidates. The bestknown election system is plurality, in which each candidate gets a point from each voter that ranks them first in their preference order, and the candidates with the highest score win.

In our setting, in addition to their preferences over the candidates, all voters are located in a metric space $\left(\mathcal{M}, d_{\mathcal{M}}\right){ }^{1}$ Each voter $v \in V$ has an associated location $x_{v} \in \mathcal{M}$ and a nonnegative distance-bound $d_{v} \in \mathbb{R}$. We also have a set of potential polling places $L \subseteq \mathcal{M}$, each place $\ell \in L$ is defined using its location. A voter is able to vote only if there is a polling place $\ell$ for which $d_{\mathcal{M}}\left(x_{v}, \ell\right) \leq d_{v}$. Formally, the winner problem for an election system $\mathcal{E}$ in this setting:

Name: $\mathcal{E}$-Geographic-Winner

Given: A set of candidates $C$, a set of voters $V$ where each $v \in V$ has a location in a metric space $x_{v} \in \mathcal{M}$ (e.g., $\mathbb{R}^{2}$ ), a preference order $\succ_{v}$, and a distance-bound to vote $d_{v}$, a set of polling places $L \subseteq \mathcal{M}$ and a candidate $p \in C$.

Question: Is $p$ a winner using election system $\mathcal{E}$ when all voters $v \in V$ within $d_{v}$ to a polling place in $L$ vote?

To compute the geographic winner for an election system $\mathcal{E}$, we must first determine which voters are within their distance-bound to a polling place. This can clearly be done in polynomial time. Thus each election system with a polynomial-time winner problem has a polynomial-time geographic winner problem. More generally:

Observation 1. For every election system $\mathcal{E}$ the corresponding $\mathcal{E}$-Geographic-Winner problem is in $\mathrm{P}^{\mathcal{E} \text {-winner. }}$

\subsection{Electoral Control}

A natural model of electoral control in geographic settings is to consider how an election chair with control of the election can select polling places to ensure their preferred outcome. It is realistic to assume that the election chair is required to place at least a specified number of polling places, since otherwise the election would be easily viewed as unfair. We present the formal definition of the constructive case below (where the goal of the election chair is to ensure a preferred candidate wins) [Bartholdi III et al., 1992], but we will also consider the destructive case (where the goal of the election chair is to ensure that a despised candidate does not win) [Hemaspaandra et al., 2007].

Name: $\mathcal{E}$-Constructive Polling Place Control

\footnotetext{
${ }^{1}$ We stress that this is not putting the voters and candidates in an "ideological" metric space, à la Schofield [2008]. This is literal physical space, which has nothing to do with the preferences.
} 
Given: A set of candidates $C$, a set of voters $V$ where each $v \in V$ has a location in a metric space $x_{v} \in \mathcal{M}$, a preference order $\succ_{v}$, and a distance to vote $d_{v}$, a set of possible polling places $L \subseteq \mathcal{M}$, a preferred candidate $p \in C$, and a parameter $k$.

Question: Does there exist a set of polling places $L^{\prime} \subseteq L$ such that $\left|L^{\prime}\right| \geq k$ and $p$ is a winner of the geographic election $\left(C, V, L^{\prime}\right)$ using the election system $\mathcal{E}$ ?

\section{The Two-Party System}

In this section we will focus on settings of a two-party system, which is quite common across democratic countries. That is, we assume $|C|=2$.

We first consider a simpler version of our problem, where the voters and polling places are on the real line, i.e., $\mathcal{M}=\mathbb{R}$. For our voting scenario, this can model situations such as the selection of polling places along a bus line in a city, where the bus line's route can be viewed as a straight line.

Theorem 2. Constructive and Destructive Polling Place Control for plurality elections over two candidates with voters located on the real line $(\mathcal{M}=\mathbb{R})$ is in $\mathrm{P}$.

ProOF. We consider the constructive case below. This algorithm can be easily adapted for the destructive case.

Let $C=\{p, r\}, V, L, p$, and $k$ be an instance of Polling Place Control where voters and polling places are located on $\mathbb{R}$. We will show that we can determine if $p$ can be made a plurality winner in the geographic setting by selecting voting locations using dynamic programming in polynomial time.

Our dynamic programming algorithm works as follows. We first order the polling places with respect to $\mathbb{R}$. We construct the dynamic programming table so that $T[a, b$, last $]$ is the maximum margin for $p$ (i.e., $\operatorname{score}(p)-\operatorname{score}(r))$ using $b$ of the first $a$ polling places (ordered from left to right along $\mathbb{R})$, where "last" is the last polling place picked. A solution to our problem is found if there is a nonnegative margin in the table for $k$ polling places. When filling out the table we can easily determine which voters within their distance to a polling place under consideration are overlapping with a polling place already picked with just the information for the last polling place picked.

We now continue, and try to see if this result holds for more complex metric mechanisms.

Theorem 3. Constructive and Destructive Polling Place Control for plurality elections with voters located in the plane $\left(\mathcal{M}=\mathbb{R}^{2}\right)$ is NP-complete for two candidates even when all voters have the same distance-bound.

PROOF. Membership of our problem in NP is easy to see.

Our voting problem locates the voters in the plane, so the most straightforward reduction will be to start with an appropriate planar NP-complete problem.

Planar Vertex Cover is NP-complete [Garey et al., 1976], even for cubic planar graphs (i.e., graphs where each vertex has degree at most three) [Garey and Johnson, 1977].

Name: Cubic Planar Vertex Cover

Given: A Cubic (i.e., maximal degree is 3 or fewer) planar graph $G=(V, E)$ and an integer $k$.

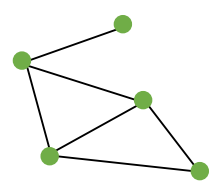

(a)

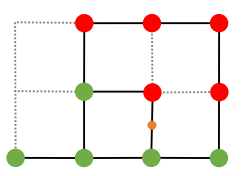

(c)

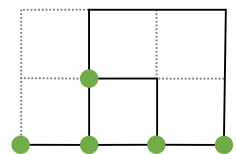

(b)

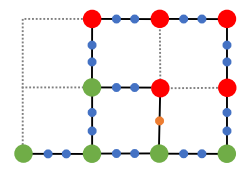

(d)
Figure 1: The process to produce $\widehat{G}$ in the proof of Lemma 4 . (a) shows our initial graph; (b) shows a graph embedded onto the grid; (c) shows the addition of nodes at grid points, and a special orange node to make each edge have even number of additional vertices; (d) shows the added pairs of vertices dividing the larger edges.

Question: Does there exist a subset of vertices $V^{\prime} \subseteq V$ such that $\left|V^{\prime}\right| \leq k$ and for each $(u, v) \in E, u \in V^{\prime}$ or $v \in V^{\prime}$ ?

It will be useful to work with this problem embedded into the grid. We will show that the following rectilinear version of Cubic Planar Vertex Cover is NP-complete. ${ }^{2}$

Name: Restricted Rectilinear Cubic Planar Vertex Cover

Given: A cubic planar graph $G=(V, E)$ embedded in a grid such that all edges are on integer gridlines and of length 1 or 1.5 , and an integer $k$.

Question: Does there exist a subset of vertices $V^{\prime} \subseteq V$ such that $\left|V^{\prime}\right| \leq k$ and for each $(u, v) \in E, u \in V^{\prime}$ or $v \in V^{\prime}$ ?

Lemma 4. Restricted Rectilinear Cubic Planar Vertex Cover is NP-complete.

Proof. Membership in NP is easy to see. Let $G=(V, E)$ and $k$ be an instance of Cubic Planar Vertex Cover.

Valiant [1981] shows that a planar graph $(V, E)$ where each vertex has maximum degree four can be drawn in polynomial time in a $O(|V|) \times O(|V|)$ grid such that each vertex has integer coordinates and the edges are comprised of line segments along the integer gridlines, and the edges do not intersect with each other. We apply this construction to our graph and then subdivide the edges by adding vertices at each intersection of integer gridlines so that each edge in this new graph has length one. If an edge is not subdivided by an even number of vertices, we add an additional vertex at the midpoint between one of the original vertices of the edge and the closest vertex we added, so that overall, every original edge has been divided by adding an even number of vertices to the edge. Now all of the edges are length 1 or $1 / 2$.

For each of the edges of length 1 , we add two additional vertices to subdivide the edge into three equal segments, i.e., one at $1 / 3$ and the other at $2 / 3$. Note that this maintains that each original edge has been divided by an even number of new vertices. We then rescale all of our edges by 3 so that

\footnotetext{
${ }^{2}$ We mention that some of our construction follows the general contours of a construction from Chan and $\mathrm{Hu}$ [2015], which shows a related geometric set covering problem to be NP-hard.
} 


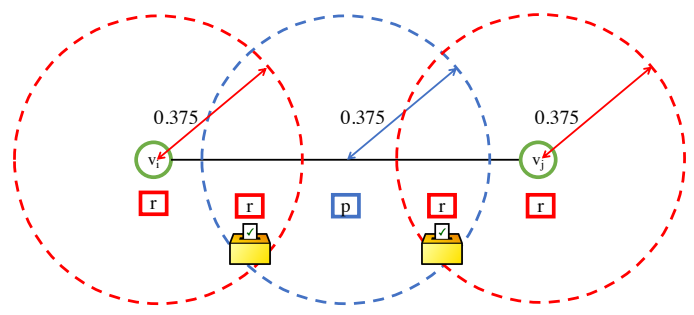

Figure 2: The added voters and ballot boxes on an edge $\left(v_{i}, v_{j}\right)$. The circles denote the voters' distance-bounds. In order not to complicate the drawing further, the distance-bounds for the $r$ voters on the edges are not shown, but they are 0.375 , thus extending past the $p$ voter on one side and a vertex on the other, but not as far as reaching another polling place.

they are all of length 1.5 or of length 1 . Denote this new graph $\widehat{G}=(\widehat{V}, \widehat{E})$ (see Figure 1$)$.

When we subdivide an edge by adding two vertices to a given graph, the size of a minimum vertex cover increases by one. Each edge in a graph has at least one of its vertices in a minimum vertex cover. When an edge is subdivided by adding two vertices, it is "replaced" with three edges. One of the outer edges must be covered by a vertex in the original minimum vertex cover. To cover the edge between the two added vertices and the other outer edge, one of the added vertices must be included in a minimum vertex cover of the updated graph.

It is straightforward to see that $G$ has a vertex cover of size at most $k$ if and only if $\widehat{G}$ has a vertex cover of size $\hat{k}=k+0.5 t$, with $t$ being the number of added vertices.

Having shown Lemma 4, we will now put it to use. Let $G=(V, E)$ and $k$ be an instance of Restricted Rectilinear Cubic Planar Vertex Cover. We now construct an instance of Constructive Polling Place Control for plurality elections (see Figure 2 for an example of a constructed edge).

- Let the set of candidates be $\{p, r\}$.

- For each vertex $v_{i} \in V$ create one voter for $r$ at that location with distance-bound 0.375 .

- For each edge $\left\{v_{i}, v_{j}\right\} \in V$ create one voter voting for $p$ located at the midpoint of the edge with distance-bound 0.375 .

- For each edge $\left\{v_{i}, v_{j}\right\} \in V$ add two polling places to $L$ : one at the midpoint between the voter for $r$ at $v_{i}$ and the voter for $p$ at the midpoint, and the other at the midpoint between the voter for $r$ at $v_{j}$ and the voter for $p$.

- At the location of every polling place, add an additional voter for $r$. This brings to a total of three voters located on each edge (without the vertices) — two supporting $r$ and one supporting $p$.

- Add one additional polling place $\hat{q}$ at a distance strictly greater than 1.5 from the constructed graph with an additional $k$ voters for $p$ located at that same location with distance-bound 0.375 .

- Let the number of polling places to have be $|E|+1$.
We will now show that $G$ has a vertex cover of size $k$ if and only if there exists a subset of at least $|E|+1$ polling places to select such that $p$ is a plurality winner in the geographic setting.

Suppose that $G$ has a vertex cover of size $k$. Let this vertex cover be $V^{\prime}$. For each vertex $v \in V^{\prime}$, select each polling place within a distance of 0.375 to $v$ if there is not already a polling place selected on that edge. Since $V^{\prime}$ is a vertex cover, we will have selected $|E|$ polling places. It is easy to see that selecting these polling places will result in $|E|$ voters for $p$ and $|E|+k$ voters for $r$ to be within their distance to a polling place. $p$ is made a winner by also selecting the polling place $\hat{q}$.

For the converse, suppose that $G$ does not have a vertex cover of size $k$. Then we will show that there is no subset of at least $|E|+1$ polling places to add such that $p$ is a plurality winner in the geographic setting. There are at most $|E|$ votes for $p$ among the polling places in $L^{\prime} \backslash \hat{q}$, and if we select two polling places on the same edge, the second only adds more votes for $r$ (specifically either one or two additional votes for $r$ ), and no votes for $p$. Assume that we have a subset $L^{\prime}$ of at least $|E|+1$ polling places such that $p$ is a plurality winner in the geographic setting. Then we know that $\hat{q}$ must be in $L^{\prime}$, and the remaining polling places selected can have at most $k$ more votes for $r$ than for $p$. Consider the remaining polling places in $L^{\prime} \backslash \hat{q}$. We know that at least $|E|$ polling places were selected, and they contribute at most $k$ votes for $r$ over $p$. However, the only polling places which do not add more $r$ votes than $p$ votes are those for which the $r$ voter located on a vertex already votes elsewhere. That is, the vertex voter overlaps for several polling places. If $p$ is a winner, this overlap must happen for at least $|E|-k$ polling places. That is, $|E|-k$ polling places did not change the balance between $p$ and $r$ and did not include the vertex $r$ voter. The $k$ that did include that voter are thus a vertex cover of $G$ of size $k$. This is a contradiction.

Notice that essentially the same reduction can be used for the destructive case. There the goal would be to ensure that $r$ does not win. And there would be $k+1$ votes for $p$ at $\hat{q}$. So then in the case where there is a vertex cover, $p$ beats $r$, and in the case where there is not a vertex cover, $r$ is a winner.

\subsection{Natural Restrictions to the Planar Case}

When we consider some natural restrictions to the overlap between choice of polling places we have several tractable cases. The simplest case is when no voter is able to vote at more than one of the polling places under consideration. In this case it is easy to see that the greedy approach of choosing the polling places in order of margin for $p$ is optimal when there are two candidates.

Theorem 5. Constructive and Destructive Polling Place Control for plurality elections over two candidates with voters located in any metric space $\mathcal{M}$ is in $\mathrm{P}$ when no voter $v \in V$ is within $d_{v}$ of more than one polling place in $L$.

Notice that this result can be extended to instances where there is a fixed number of polling places that share voters in common. Due to space restrictions, the proof of the theorem below has been omitted. 
Theorem 6. Constructive and Destructive Polling Place Control for plurality elections over two candidates with voters located in a metric space $\mathcal{M}$ is in $\mathrm{P}$ when the number of overlapping polling places is fixed.

It is natural to wonder if this result can be pushed any further. For example, for a fixed parameter $\ell$, each voter is within their distance to at most $\ell$ polling places. However, the construction from the proof of Theorem 3 shows hardness for the case of $\ell=3$. This leaves open the question of what happens for the case of $\ell=2$. In Example 7 below we show that the obvious greedy approach will not solve this case.

Example 7. Examine this instance of polling place control. Let $C=\{p, r\}$ be the set of candidates, let the set of polling places be $L=\{A, B, C\}$ with $A$ located at $(1,1)$, B located at $(2,2)$, and $C$ located at $(2.5,1)$. Let there be the following voters all with distance 1.

20 voters for $p$ at $(1.5,0.5)$ that can vote at $A$ or $C$.

30 voters for $p$ at $(1.5,1.5)$ that can vote at $A$ or $B$.

35 voters for $r$ at $(2.5,1.5)$ that can vote at $B$ or $C$.

51 voters for $r$ at $(1,1)$ that can vote at $A$.

5 voters for $r$ at $(2,2)$ that can vote at $B$.

Let $k=2$ and the preferred candidate be $p$.

The margin for $p$ at $A$ is -1 , at $B$ is -10 , and at $C$ is -15 . So the greedy approach will choose A. After choosing $A$, the margin for $p$ at $B$ is -40 and at $C$ is -35 . And this algorithm would return that there is no way to allocate at least 2 polling places such that $p$ wins. However, if we instead choose the polling places $B$ and $C, p$ wins.

This greedy approach fails to find a solution when one exists due to the overlap of voters between polling places, and so it cannot even be used as an approximation.

\section{Multi-Party System}

Unlike in the case of different distance-bounds for voters, when we move to the case of more than two candidates, polling place control for plurality elections becomes NPcomplete even when voters can vote at most at one location.

When voters can vote at most at one location, the mapping to the metric space is trivial and we are instead left with considering how to add groups of voters to an initially empty set of voters to ensure that a preferred candidate wins. We show the following hardness result for the case of an unbounded number of candidates where the voting locations do not serve any of the same voters as another (that is, making the problem easier, as can be seen in the two-party case above).

Theorem 8. Constructive Polling Place Control for plurality elections is NP-complete for multiple candidates even when voters can vote at most at one location and $\mathcal{M}=\mathbb{R}$.

Proof. Membership in NP is easy to see. We show NPhardness by a reduction from Exact Cover by 3-Sets [Karp, 1972].

Name: Exact Cover by 3-Sets

Given: A set $B=\left\{b_{1}, \ldots, b_{3 k}\right\}$, and a collection $\mathcal{S}=$ $\left\{S_{1}, \ldots, S_{n}\right\}$ of three-element subsets of $B$.

Question: Does there exist a subcollection $\mathcal{S}^{\prime}$ of $\mathcal{S}$ such that every element of $B$ occurs in exactly one member of $\mathcal{S}^{\prime}$ ?
Given an instance of Exact Cover by 3-Sets, $B=$ $\left\{b_{1}, \ldots, b_{3 k}\right\}$ and $\mathcal{S}=\left\{S_{1}, \ldots, S_{n}\right\}$ such that each $S_{i}=$ $\left\{b_{i 1}, b_{i 2}, b_{i 3}\right\}$, we construct the following instance of polling place control.

Let the set of candidates be $C=B \cup\{p\}$. In this construction, all voters will have distance-bound $1 / 2$. For each $S_{i} \in \mathcal{S}$, create a polling place at $(i, 0)$ with $k-2$ votes for each $b_{i}$, with an additional $k$ votes for $b_{i 1}, b_{i 2}$, and $b_{i 3}$ (so each of these candidates has $2 k-2$ voters at this polling place). Add another polling place at $(n+1,0)$ with $k^{2}-k$ votes for $p$. Let the preferred candidate be $p$, and let the bound on polling places to select be at least $k+1$.

Suppose there exists an exact cover $\mathcal{S}^{\prime}$. For each $S_{i} \in \mathcal{S}^{\prime}$, add the polling place at $(i, 0)$. Then each candidate $b_{i}$ has score exactly $(k-2) k+k=k^{2}-k$. When the polling place at $(n+1,0)$ is added, $p$ is a winner with score $k^{2}-k$.

If there is a way to make $p$ win by choosing at least $k+1$ polling places then there must exist an exact cover. We know that the polling place at $(n+1,0)$ must have been selected, since it is the only polling place where $p$ gets any votes. The remaining polling places selected must give each $b_{i}$ candidate at most $k^{2}-k$ votes and so exactly $k$ of the polling places in $L \backslash(n+1,0)$ were selected (otherwise, there is a candidate which appears in at least two sets, getting $2 k-2$ voters in two different polling places). The $k$ polling places other than $(n+1,0)$ must correspond to an exact cover, otherwise some candidate $b_{i}$ has score greater than $k^{2}-k$.

\subsection{Inapproximability}

Consider the optimization version of polling place control where we seek to maximize the number of polling places selected. This problem exhibits nonmonotonicity as for a given set of polling places $L$ that ensures a candidate $p$ wins it is not always the case that $p$ wins when a subset of $L$ is selected.

Theorem 9. It is NP-hard to approximate by any factor the maximum number of polling places to select such that $p$ is a plurality winner in the geographic setting for multiple candidates. This holds even when voters can vote at most at one location and $\mathcal{M}=\mathbb{R}$.

The proof adapts the construction from Theorem 8 (by adding a candidate and spreading around $p$ 's voters) to show that $p$ wins with a nonzero set of polling places if and only if there is an exact cover. Thus, any multiplicative approximation reveals the answer to the NP-hard problem. A similar approach to showing inapproximability was used by Caragiannis et al. [2012] to prove the inapproximability of Young score, another voting problem that exhibits nonmonotonicity as an optimization problem.

\subsection{Standard Control Actions in the Geographic Setting}

Our focus has been to explore how an election chair with control over the selection of voting locations can ensure their preferred outcome in a geographic election. However, as previously mentioned, this geographic model for elections is quite natural and standard models of control (and many other election problems) can be considered in this setting. 
It is easy to see that NP-hardness results from the standard setting for control by adding/deleting/partitioning candidates/voters in both the constructive and the destructive cases are inherited to the corresponding actions of control by adding/deleting/partitioning candidates/voters for geographic elections. First, we show the existing problems' results extend to our new domain (proofs omitted due to space constraints).

Observation 10. Each standard constructive and destructive control action polynomial-time many one reduces to the control action in the geographic setting.

As a result of Observation 10, for a given election system, if a standard control action is NP-hard, it remains NP-hard in the geographic setting. At first glance it may seem that the structure of the geographic setting may be able to realize an increase in the complexity of polynomial-time control actions. However, this is not the case.

Observation 11. Each standard constructive and destructive control action in the geographic setting polynomial-time many one reduces to the standard control action.

\subsection{Additional Control Actions}

We can also define additional new natural models for control in the geographic setting. For example, consider the scenario where an agent can change the distance-bound for a subcollection of the voters to ensure their preferred outcome. We wish to stress that while in a regular voting setting one might scoff at directly bribing voters as a rare occurrence (except as campaigning), in our setting, changing the distance bound for voters is easily done with perfectly legal means-organize a ride, or a bus, or any other form of transport to help voters reach their polling places. For preventing voters to vote, a candidate can cause traffic delays with rallies (or politicallydevised traffic jams [Zernike, 2015]):

Name: $\mathcal{E}$-Constructive Control by Distance-Bound Change Given: A set of candidates $C$, a set of voters $V$ where each $v \in V$ has a location in a metric space $x_{v} \in \mathcal{M}$ (e.g., the plane, $\mathbb{R}^{2}$ ), a preference order $\succ_{v}$, and a distance-bound to vote $d_{v}$, a set of polling places $L \subseteq \mathcal{M}$ and a candidate $p \in$ $C$, and a budget $k$.

Question: Do there exist subcollections of voters $V^{\prime} \subseteq V$ and $W^{\prime} \subseteq V$ with $V^{\prime} \cap W^{\prime}=\emptyset$ such that

$$
\begin{aligned}
\left(\sum_{v_{i} \in V^{\prime}}\left(d_{v_{i}}-\min \left(d_{v_{i}}, d_{\mathcal{M}}\left(x_{v_{i}}, L_{v_{i}}\right)\right)+\epsilon\right)\right)+ & \\
\left(\sum_{v_{i} \in W^{\prime}}\left(\max \left(d_{v_{i}}, d_{\mathcal{M}}\left(x_{v_{i}}, L_{v_{i}}\right)\right)-d_{v_{i}}\right)\right) & \leq k
\end{aligned}
$$

for some $\epsilon>0,{ }^{3} L_{v_{i}}$ is the location of the polling place nearest $v_{i}$ and $p$ is a winner using election system $\mathcal{E}$ of the geographic election $(C, \widehat{V}, L)$ where $\widehat{V}$ consists of the voters from $V$ with updated distance-bounds for voters in $V^{\prime}$ and $W^{\prime}$ ?

In the problem above, the difference between a voter's distance-bound and the distance to the closest polling place

\footnotetext{
${ }^{3}$ Practically, it is as small as one wishes. It is simply needed to make the distance of voter $v_{i}$ just a bit smaller than will make it able to reach its ballot box.
}

can be viewed as their "price" for participating in the election. We can show this problem to be in $\mathrm{P}$ for plurality elections by a reduction to multimode priced control by adding voters and deleting voters introduced by Miasko and Faliszewski [2016].

Name: $\mathcal{E}$-Priced Constructive Control by Adding Voters and Deleting Voters

Given: An election $(C, V)$, a set of unregistered voters $W$, cost function cost for each voter in $V \cup W$, a candidate $p \in C$, and a budget $k$.

Question: Do there exist subcollections $V^{\prime} \subseteq V$ and $W^{\prime} \subseteq$ $W$ such that $\operatorname{cost}\left(V^{\prime} \cup W^{\prime}\right) \leq k$ and $p$ is a winner of the election $\left(C,\left(V \backslash V^{\prime}\right) \cup W^{\prime}\right)$ using election system $\mathcal{E}$ ?

Theorem 12. Constructive Control by Distance-Bound Change for plurality elections is in $\mathrm{P}$.

Proof omitted due to space constraints.

More natural control actions to consider in future work are integrating control by selecting polling places into the framework of multimode electoral control introduced by Faliszewski et al. [2011]. Briefly, multimode control considers the scenario where the election chair uses multiple control actions to achieve their goal. One example is the control by adding voters and deleting voters mentioned above for the priced setting. It is natural to consider that an election chair that is able to select polling places would also be able to control the structure of the election in other ways.

\section{Discussion and Future Work}

We introduced a new model for studying election problems that takes into account geographic information. We examined the complexity of electoral control by selecting voting locations in this geographic setting. We compared how the complexity is affected by different parameters such as the two-party setting and settings with an unbounded number of candidates, and the cases where voters and polling places are placed on the line vs. being placed on the plane. Furthermore, we linked this setting with some of the existing problems in voting complexity in the nongeographic setting.

There are many different avenues for future work, as noted at the end of the previous sections. A specific open problem for future work is the complexity of polling place control when voters can vote at most at two locations. Beyond that, further exploring how existing problems in election manipulation and control change once the geographic element is added is an interesting idea, as well as introducing new models to capture natural scenarios in this setting. A more radical direction is to re-interpret the distance-bounds $d_{v}$ as a statistical measure, indicating probability of voting at a location of particular distance. This allows for richer settings, and more complex problems and analysis, and combines more deeply with geographic (urban/rural) considerations.

\section{Acknowledgements}

The authors thank Edith Hemaspaandra and the anonymous reviewers for helpful comments. Research was done in part while Zack Fitzsimmons was on research visits to BenGurion University and Rensselaer Polytechnic Institute. 


\section{References}

[Bachrach et al., 2016] Yoram Bachrach, Omer Lev, Yoad Lewenberg, and Yair Zick. Misrepresentation in district voting. In Proc. of IJCAI-16, pages 81-87, July 2016.

[Bartholdi III et al., 1992] John Bartholdi III, Craig Tovey, and Michael Trick. How hard is it to control an election? Mathematical and Computer Modeling, 16(8/9):2740, 1992.

[Bervoets and Merlin, 2012] Sebastian Bervoets and Vincent Merlin. Gerrymander-proof representative democracies. International Journal of Game Theory, 41:473-488, 2012.

[Borodin et al., 2018] Allan Borodin, Omer Lev, Nisarg Shah, and Tyrone Strangway. Big city vs. the great outdoors: Voter distribution and how it affects gerrymandering. In Proc. of IJCAI-18, pages 98-104, July 2018.

[Bulteau et al., 2015] Laurent Bulteau, Jiehua Chen, Piotr Faliszewski, Rolf Niedermeier, and Nimrod Talmon. Combinatorial voter control in elections. Theoretical Computer Science, 589(19):99-120, 2015.

[Caragiannis et al., 2012] Ioannis Caragiannis, Jason Covey, Michal Feldman, Christopher Homan, Christos Kaklamanis, Nikos Karanikolas, Ariel Procaccia, and Jeffrey Rosenschein. On the approximability of Dodgson and Young elections. Artificial Intelligence, 187:31-51, 2012.

[Chan and Hu, 2015] Timothy Chan and Nan Hu. Geometric red-blue set cover for unit squares and related problems. Computational Geometry, 48(5):380-385, 2015.

[Cohen-Zemach et al., 2018] Amittai Cohen-Zemach, Yoad Lewenberg, and Jeffrey Rosenschein. Gerrymandering over graphs. In Proc. of AAMAS-18, pages 274-282, July 2018.

[Erdélyi et al., 2015] Gábor Erdélyi, Edith Hemaspaandra, and Lane Hemaspaandra. More natural models of electoral control by partition. In Proc. of ADT-15, pages 396-413, September 2015.

[Faliszewski and Rothe, 2016] Piotr Faliszewski and Jörg Rothe. Control and bribery in voting. In Felix Brandt, Vincent Conitzer, Ulle Endriss, Jérôme Lang, and Ariel Procaccia, editors, Handbook of Computational Social Choice, pages 146-168. Cambridge University Press, New York, 2016.

[Faliszewski et al., 2009] Piotr Faliszewski, Edith Hemaspaandra, and Lane Hemaspaandra. How hard is bribery in elections? Journal of Artificial Intelligence Research, 35:485-532, 2009.

[Faliszewski et al., 2011] Piotr Faliszewski, Edith Hemaspaandra, and Lane Hemaspaandra. Multimode attacks on elections. Journal of Artificial Intelligence Research, 40:305-351, 2011.

[Faliszewski et al., 2015] Piotr Faliszewski, Edith Hemaspaandra, and Lane Hemaspaandra. Weighted electoral control. Journal of Artificial Intelligence Research, 52:507-542, 2015.
[Feldman et al., 2016] Michal Feldman, Amos Fiat, and Iddan Golomb. On voting and facility location. In Proc. of EC-16, pages 269-286, July 2016.

[Garey and Johnson, 1977] Michael Garey and David Johnson. The rectilinear Steiner tree problem is NP-complete. SIAM Journal on Applied Mathematics, 32(4):826-834, June 1977.

[Garey et al., 1976] Michael Garey, David Johnson, and Larry Stockmeyer. Some simplified NP-complete graph problems. Theoretical Computer Science, 1(3):237-267, 1976.

[Hemaspaandra et al., 2007] Edith Hemaspaandra, Lane Hemaspaandra, and Jörg Rothe. Anyone but him: The complexity of precluding an alternative. Artificial Intelligence, 171(5-6):255-285, 2007.

[Karp, 1972] Richard Karp. Reducibility among combinatorial problems. In Raymond Miller and James Thatcher, editors, Complexity of Computer Computations, pages 85103, 1972.

[Lev and Lewenberg, 2019] Omer Lev and Yoad Lewenberg. "Reverse gerrymandering": a decentralized model for multi-group decision making. In Proc. of AAAI-19, pages 2069-2076, January-February 2019.

[Lewenberg et al., 2017] Yoad Lewenberg, Omer Lev, and Jeffrey Rosenschein. Divide and conquer: Using geographic manipulation to win district-based elections. In Proc. of AAMAS-17, pages 624-632, May 2017.

[Miasko and Faliszewski, 2016] Tomasz Miasko and Piotr Faliszewski. The complexity of priced control in elections. Annals of Mathematics and Artificial Intelligence, 77(3-4):1-26, August 2016.

[Nichols, 2018] Mark Nichols. Closed voting sites hit minority counties harder for busy midterm elections. USA Today, October 302018.

[Pegden et al., 2017] Wesley Pegden, Ariel Procaccia, and Dingli Yu. A partisan districting protocol with provably nonpartisan outcomes. Technical Report arXiv:1710.08781 [cs.GT], arXiv.org, October 2017.

[Reid, 2018] Tim Reid. Georgia candidates decry plan to close voting sites in mostly black county. Reuters, August 212018.

[Schofield, 2008] Norman Schofield. The Spatial Model of Politics. Number 95 in Routledge Frontiers of Political Economy. Routledge, New York, 2008.

[Valiant, 1981] Leslie Valiant. Universality considerations in VLSI circuits. IEEE Transactions on Computers, 100(2):135-140, 1981.

[van Bevern et al., 2015] René van Bevern, Robert Bredereck, Jiehua Chen, Vincent Froese, Rolf Niedermeier, and Gerhard Woeginger. Network-based vertex dissolution. SIAM Journal on Discrete Mathematics, 29(2):888-914, 2015.

[Zernike, 2015] Kate Zernike. The bridge scandal, explained. New York Times, May 12015. 